\title{
Del trabajo de referencia hacia la alfabetización informativa: La evolución del papel educativo del bibliotecario *
}

\author{
Bernadete Campello **
}

Artículo recibido:

9 de noviembre de 2009.

Artículo aceptado:

16 de marzo de 2010.

\section{RESUMEN}

Se describe la evolución del papel educativo del bibliotecario desde la aparición del trabajo de referencia hasta el surgimiento del concepto de alfabetización informativa y su asimilación por la biblioteconomía. Y demuestra que la idea de alfabetización informativa estaba ya incrustada en las prácticas relacionadas con el trabajo de referencia y de educación de usuarios. Sin embargo el nuevo concepto trajo consigo la expansión de la función educativa del bibliotecario. Tomando en

* $\quad$ Artículo basado en la tesis de doctorado defendida por la autora en el Programa de Posgrado en Ciencia de la Información de la UFMG: Campello, B. Alfabetización informativa en Brasil: prácticas educativas de bibliotecarios en escuelas de enseñanza básica. 2009. Tesis (Doctorado en Ciencia de la Información). Escuela de Ciencia de la Información, Universidad Federal de Minas Gerais, Belo Horizonte, 2009.

** Escola de Ciência da Informação da UFMG. Belo Horizonte Brasil. campello@eci. ufmg.br

INVESTIGACIÓN BIBLIOTECOLÓGICA, Vol. 24, Núm. 50, enero/abril, 2010, México, ISSN: 0187-358X. pp. 83-108 
cuenta la responsabilidad que el bibliotecario asume actualmente, de ayudarles a las personas a que aprendan a buscar y usar la información, se concluye que tendrá no sólo que buscar una mejor comprensión del concepto de alfabetización informativa sino también de modificar su práctica educativa.

Palabras clave: Alfabetización informativa; Bibliotecario; Biblioteca.

\begin{abstract}
From reference work to information literacy: evolution of the librarians' educational role Bernadete Campello

The evolution of the educational role of librarians, since the appearance of reference work to the emergence of information literacy and its assimilation by the library profession, is described. The idea of information literacy is shown to be intrinsically embedded related to reference work practices and user education. However, the new concept brought about the widening of the educational role of the librarian. In view of the responsibility assumed currently by librarians -to help people learn how to search and use informationa better understanding of the concept of information literacy is necessary, as well as changes in educational practice.
\end{abstract}

Keywords: Information literacy; Librarian; Library

\title{
INTRODUCCIÓN
}
A
partir de las ideas de Ortega y Gasset sobre la misión del bibliotecario, se elabora aquí una descripción sobre la evolución del papel educativo de este profesional, con el propósito de comprender de qué manera el concepto de alfabetización informativa se venía insertando en la biblioteconomía como uno de los fundamentos para desempeñar ese papel. Pretendemos elucidar el recorrido educativo del bibliotecario a partir de las prácticas de auxilio al usuario, formalizadas con el trabajo de referencia, hasta la asimilación del 
concepto de alfabetización informativa, que actualmente propone que el bibliotecario asuma un papel activo en el proceso de enseñarles a las personas a que aprendan cómo buscar y usar la información Mi intención es mostrar que la idea de alfabetización informativa ya hacía parte de las prácticas relacionadas con el servicio de referencia y de educación de usuarios, y que la nueva denominación contribuye a ampliar la función educativa del bibliotecario.

\section{LA MISIÓN DEL BIBLIOTECARIO}

Las condiciones del surgimiento y de evolución de los diferentes papeles del bibliotecario fueron analizadas desde hace sesenta años por el filósofo español Ortega y Gasset en una conferencia realizada en 1935, durante el $2^{\circ}$ Congreso Mundial de Bibliotecas y Bibliografía, en Madrid. El autor se basó en la suposición de que al ejercer una profesión, la persona se compromete a hacer lo que la sociedad necesita, con lo cual renuncia a su libertad individual de elección y pasa a actuar en una perspectiva colectiva. Cuando ejerce su práctica, el profesional no está ejerciendo algo individual sino ocupando un lugar social, que es demandado y sostenido por la sociedad, y no sólo por la vocación ocasional de uno u otro individuo (Ortega y Gasset, 1967, p. 68).

Ortega y Gasset argumentaba que las necesidades que la sociedad manifiesta no son fijas sino variables y evolutivas, esto es, históricas. Así, la función del bibliotecario variaría de acuerdo con la necesidad social que la profesión debe atender y esa necesidad estaría relacionada con el libro. Según el autor, parece incuestionable que "el quehacer del bibliotecario haya variado siempre en rigurosa función de lo que el libro significaba como necesidad social” (Ortega y Gasset, 1967, p. 70). La profesión se originó pues con el surgimiento del libro y ha venido evolucionando junto con él, y alejándose de su noción restricta de instrumento de revelación o código, tornándose, a la vez, en algo necesario y útil para la sociedad, y aun esa necesidad y utilidad van asumiendo contornos diferentes, dependiendo de las demandas sociales de cada momento. Hasta el siglo XV la función del bibliotecario se relacionaba sobre todo con la necesidad de adquirir libros para las bibliotecas, pues había pocos. Con el Renacimiento la demanda por el libro aumentó, pues el periodo se caracterizaba por la necesidad de producir libros, lo que llevó a la invención de la imprenta. Ya al inicio del siglo XIX, el libro no era más un objeto escaso; su producción se hacía en mayor cantidad y eso tornó necesaria su organización en las bibliotecas, su catalogación; una demanda que, según Ortega y Gasset, estaba relacionada con la materialidad del libro. En cuanto 
al aspecto intelectual, su contenido, había necesidad de fomentar la lectura y de buscar lectores (Ortega y Gasset, 1967, p. 74), pues con la Revolución Francesa y la creación de los estados democráticos el libro se tornaba imprescindible, ya que la democracia no podría sobrevivir sin un "gran repertorio de ideas" (Ortega y Gasset, 1967, p. 77). Hasta aquel momento el libro era un instrumento sobre todo útil para solucionar el problema de conservar las ideas, pero a partir de la segunda mitad del siglo XIX, el propio libro se volvió un problema debido a su cantidad. Se amplió así de nuevo la función del bibliotecario, que pasó a ser más compleja y a incluir la organización colectiva de la producción del libro, la búsqueda de técnicas bibliográficas más eficientes y, finalmente, la orientación de los lectores a través de la selva de libros. Esa última es la nueva función del bibliotecario, superior a las anteriores, según Ortega y Gasset, pues se aleja del libro como objeto e introduce la función de orientar al lector. Hasta ese momento, de acuerdo con el autor, el libro constituía una necesidad marcada por un signo positivo, pero la producción excesiva, característica que se preanunciaba al inicio del siglo XX, transformaba el libro en signo negativo. Así, la demanda por una profesión relacionada con el libro tenía que ver con la necesidad de "orientar al lector no especializado en la 'selva selvaggia' de los libros". Ya no era sólo suficiente la simple administración del objeto. El libro como objeto puede solamente conservar las palabras, pero la lectura crítica puede transformar en legítimas las ideas contenidas en un libro; por lo tanto, el lector, "antes de leer el libro ha pensado por sí mismo el tema y conoce sus veredas" (Ortega y Gasset, 1967, p. 98).

Así, en la Misión del Bibliotecario, Ortega y Gasset delineó los tres papeles centrales del bibliotecario, que en la actualidad pueden ser explicitados así: (1) formar acervos estructurados y coherentes; (2) organizar esos acervos, como forma de potencializar su uso y (3) ayudar a los usuarios a que aprendan con base en esos acervos. Ese último representa lo que estamos aquí denominando la función educativa del bibliotecario.

\section{LA CONSOLIDACIÓN DEL PAPEL EDUCATIVO}

La consolidación del papel educativo del bibliotecario ocurrió a lo largo del tiempo, en un trayecto fortalecido tanto por las prácticas implementadas en las bibliotecas como por las investigaciones académicas, que posibilitaron la construcción de un aparato teórico/conceptual que ha sostenido esas prácticas y con ello reforzado la necesidad de participar efectivamente en el proceso de enseñanza-aprendizaje. 
En esta evolución de las prácticas de la biblioteconomía, la atención al usuario se formalizó inicialmente en el trabajo de referencia y, con la creación de éste, la función educativa de la biblioteca se hizo más explícita. El trabajo de referencia designa ese proceso de asistencia directa y personal que hace el bibliotecario con el lector que busca la información, lo que por tanto presupone la intervención activa de ese profesional en el proceso de búsqueda de información.

\section{Trabajo de referencia}

El trabajo de referencia surgió dentro del movimiento de la biblioteca pública en los Estados Unidos que se desarrolló paralelamente a la moción en favor de la popularización de la educación en aquel país, ${ }^{1}$ cuando se emprendió el esfuerzo para mejorar los índices de alfabetización de la población, lo que trajo como consecuencia el aumento de usuarios en las bibliotecas públicas. Eran, mientras tanto, lectores diferentes de aquellos que la biblioteca usualmente recibía (Tyckoson, 1997, p. 4-5) y constituían una categoría de usuarios que prácticamente desconocían el funcionamiento de la biblioteca, y que no tenían familiaridad con la información escrita ni con las fuentes de información. Se tornaba por lo tanto necesario que las bibliotecas se prepararan para proporcionarles a esas personas, que vivían sobre todo en la oralidad, un contacto más estrecho con los artefactos característicos del mundo alfabetizado.

El trabajo de referencia fue la solución práctica encontrada por los bibliotecarios para solucionar el problema y durante algún tiempo se desarrolló de manera casual. Su formalización tuvo inicio durante el primer congreso de la American Library Association (ALA), en 1876, cuando el bibliotecario Samuel Sweet Green, de la Worcester Free Public Library, presentó un trabajo intitulado The Desirableness of Establishing Personnal Intercourse and Relations Between Librarians and Readers in Popular Libraries. La expectativa del autor era que los bibliotecarios les enseñaran a los usuarios a usar la biblioteca, respondieran las preguntas de los lectores y los auxiliaran a seleccionar buenos libros. Esa fue la primera vez que el trabajo de referencia se discutió en el ámbito de la profesión (Tyckoson, 1997, p. 4-5).

Poco a poco el trabajo de referencia se fue sistematizando. Era practicado en niveles diversos de complejidad acompañando la evolución del aparato

1 Hasta la mitad del siglo XVIII la mayoría de las bibliotecas norteamericanas estaban relacionadas con las organizaciones privadas. Con la demanda por educación por parte de las clases populares, los liderazgos políticos percibieron la necesidad de contar con bibliotecas que funcionaran como centros de cultura para las comunidades. Hubo entonces un movimiento para la creación de bibliotecas públicas, que coincidió con el inicio del movimiento de educación popular en el país (Tyckoson, 1997, p.4). 
bibliográfico y la diversificación creciente de las demandas de los usuarios (Shera, 1973, pp. 198-199). El primer nivel de esto consistía en el auxilio que ayudaba al usuario a encontrar un determinado material en la biblioteca, ayuda que se hacía necesaria cuando el usuario desconocía el funcionamiento y la organización del acervo.

En un segundo nivel el trabajo de referencia se presentaba como ayuda para solucionar pequeños problemas de búsqueda de informaciones que no estaban específicamente representadas en el sistema. Serían seguramente hechos, datos y/o ilustraciones necesarias para completar un trabajo, las cuales normalmente demandarían para su localización un mayor grado de conocimiento de las fuentes de información.

El tercer nivel correspondía al trabajo del bibliotecario relacionado con la resolución de problemas complejos de información, y sería el considerado como más representativo de la dimensión verdaderamente profesional de la biblioteconomía. Aquí se visualizan dos vertientes para el trabajo de referencia. La primera es la vertiente educativa: en ese caso el bibliotecario ayudaría al usuario a entender el funcionamiento de la biblioteca y de las fuentes de información adecuadas para atender a sus necesidades. Se nota que, a pesar de proponerse intervenir en el proceso de búsqueda de información, ésta todavía se restringía a ayudar en la localización de los recursos informativos, y que esto ocurría principalmente en bibliotecas públicas, escolares y universitarias. La segunda vertiente, en la que el bibliotecario se encargaba de encontrar la información para el usuario, ocurría predominantemente en bibliotecas especializadas y de investigación.

El trabajo de referencia es considerado por algunos autores como espacio para la acción educativa del bibliotecario, si extrapolamos la simple orientación para localizar informaciones y la transformamos en una intervención didáctica. Así, en el ámbito del trabajo de referencia:

el bibliotecario puede ser visto como un bibliotecario-profesor, conviviendo con usuarios-alumnos. La biblioteca puede ser concebida como una escuela sin paredes, sin currículum y contenidos establecidos, con aulas sin número definido de alumnos y el bibliotecario de referencia visto como el coordinador del proceso de formación para proporcionar y orientar el uso de la información en el límite del conocimiento producido y registrado, a partir de la experiencia anterior del alumno y de su necesidad de formación. La palabra del usuario-alumno (su necesidad, su interés, su dificultad, su cuestión, su problema) inicia la clase. Para cada alumno, su clase y su contenido. Para el profesor, cada clase impartida, una clase compartida. Los dos se forman, intermediados por el conocimiento registrado (Martucci, 2000, p. 103). 
Representando el eslabón más evidente entre el usuario y los registros del conocimiento (o el espacio por excelencia para la ejecución de la función educativa del bibliotecario) el trabajo de referencia se perfeccionó gradualmente para asimilar los cambios por los cuales vienen pasando las bibliotecas (Frank et al., 1999). William Katz (1924-2004), profesor de la School of Information Science and Policy, State University of New York at Albany (USA) sistematizó esos cambios en su obra Introduction to Reference Work. Allí explica los procesos básicos del trabajo de referencia en todos sus aspectos, y presenta las principales fuentes de información en las que se basa el trabajo del bibliotecario. ${ }^{2}$

El hecho de que el alcance del trabajo de referencia estuviera limitado a los usuarios que buscaban ayuda del bibliotecario y a la necesidad de encontrar nuevas maneras de satisfacer las demandas informativas de una clientela que se expandía en búsquedas de educación formal, llevó al surgimiento de la educación de usuarios, servicio por medio del cual la biblioteca alcanzó un número mayor de usuarios al mismo tiempo que buscaba satisfacer más específicamente sus necesidades de información. Así, la educación de usuarios amplió la acción educativa de la biblioteca, pues a diferencia del trabajo de referencia -en donde el bibliotecario se ponía a disposición para responder las preguntas de los lectores- pues su característica es proactiva, y sale al encuentro del usuario, por medio de cursos, visitas guiadas y otras acciones planeadas de enseñanza acerca del uso de la biblioteca y sus recursos.

\section{Educación de usuarios}

Como la evolución de esa práctica ocurrió en los Estados Unidos, se hace evidente que los practicantes del área trabajaron asuntos relacionados con la enseñanza-aprendizaje. Fue al inicio de la década de 1950 cuando la educación de usuarios adquirió un contorno más definido en aquél país. En los países de lengua inglesa hoy la llaman library instruction o bibliographic instruction $\mathrm{y}$, sin duda, el término define con precisión su objetivo más tradicional: instruir o educar al lector en el uso de la biblioteca y de la colección, entrenándolo para meter las manos en fuentes de información consideradas como apropiadas y relevantes para el aprendizaje de determinados tópicos del programa escolar. Esa fase inicial de la educación de usuarios se caracterizaba como un "enfoque en la colección"(Stripling, 1996, p. 633) o un "abordaje de la fuente" (Kuhlthau, 1996, p. 11), y reflejaba la limitación de los objetivos que serían alcanzados.

2 Ver detalles sobre Introduction to Reference Work, en: http://www.mhhe.com/socscience/ katz/ 
En la década siguiente la función educativa de la biblioteca escolar se expandió al percibirse que la enseñanza del uso de los materiales de la biblioteca tendría que hacerse bajo la perspectiva de los contenidos curriculares. Ese abordaje fue nombrado "foco en el programa" (Stripling, 1996, p. 634) o "abordaje guía" (Kuhlthau, 1996, p. 11), en el sentido de que se pretendía conducir al estudiante en su búsqueda de información, en una secuencia de pasos que lo ayudara a entender la relación entre las diversas fuentes de la biblioteca. El énfasis estaba en una secuencia preestablecida de etapas, que básicamente tomaba en cuenta la manera de localizar y usar las fuentes para responder a una determinada pregunta y representaba un avance con relación al abordaje anterior al proponer una estrategia más abarcadora para usar los recursos de la biblioteca. El foco no era más la enseñanza de determinada fuente, sino la metodología que buscaba resaltar la utilidad de la colección para todas las áreas del currículo escolar. Aun así, se nota que permanecía la perspectiva orientada hacia la colección de la biblioteca.

En esa época, la denominación de biblioteca escolar (school library) en los Estados Unidos empezaba a cambiar hacia media center, reflejando la importancia que la escuela les confería a los recursos audiovisuales, y supuestamente transformaba a la biblioteca en un espacio diferente al expandir sus recursos y oportunidades de aprendizaje. La función de la biblioteca seguía siendo ayudar a los alumnos a usar efectivamente los recursos informativos, pero surgió la idea de que el bibliotecario debería tener un papel activo en la enseñanza de capacidades de información y que éstas deberían estar integradas al currículum (Craver, 1986, p. 186). Se proponía un abordaje diferente para la educación de usuarios, que Kuhlthau (1996, p. 11) llamó: "abordaje del proceso". En los abordajes anteriores no se enfatizaba el proceso de pensamiento lógico, la evaluación de las fuentes o la enseñanza de habilidades que involucraran la solución de problemas. El nuevo abordaje privilegiaba el uso, la interpretación y la comprensión de los significados de la información, los cuales eran considerados como evidencias a ser examinadas para responder a cuestionamientos, y la investigación bibliográfica no consistía simplemente en el descubrimiento de hechos (Kuhlthau,1996, pp. 11-12).

En la década de 1970, la clase bibliotecaria empezaba a proponer una función pedagógica más activa, y el bibliotecario debería participar en el planeamiento curricular y en discusiones con los profesores acerca de los recursos que serían los más adecuados para cada unidad de enseñanza. La biblioteca pasaba a involucrarse directamente en el desarrollo del currículo y en el proceso de enseñanza- aprendizaje (Craver, 1986, p. 187).

Para la década de 1980 se buscó definir con más claridad la función educativa del bibliotecario y se defendió la colaboración entre profesores, dirigentes 
escolares y bibliotecarios, con el fin de planear el programa de la biblioteca de acuerdo con las necesidades específicas de la escuela (Craver, 1986, p. 189). Esto definió tres papeles para el bibliotecario en la escuela: ser experto en información, profesor y consejero didáctico (instructional consultant). Como experto en información, él desarrollaría actividades específicas de la biblioteca en su perspectiva de almacén de información —-selección, adquisición, organización y divulgación del acervo - que proporcionaría el acceso físico a los recursos informativos. Como profesor, el bibliotecario enseñaría no solamente habilidades o capacidades que estaba tradicionalmente enseñando (localizar y recuperar información), sino que también se involucraría en el aprendizaje de competencias o capacidades para pensar críticamente, leer, oír y ver. Como consultor o consejero didáctico el bibliotecario debería integrar el programa de la biblioteca al currículo escolar, colaborar en el periodo de enseñanza-aprendizaje y asesorar en cuanto el planeamiento y la implantación de actividades curriculares.

Las dos últimas funciones definidas sentaban las bases para que apareciera el concepto de alfabetización informativa, que actualmente fundamenta el trabajo educativo del bibliotecario en varios países y representa el movimiento que consiguió unir la clase bibliotecaria en la búsqueda de mayor visibilidad para jugar su papel pedagógico.

\section{Alfabetización INFORMATiva}

El término "alfabetización informativa" (information literacy) fue usado por primera vez en la década de 1970, para caracterizar las competencias o capacidades necesarias para usar las fuentes electrónicas de información que se estaban produciendo en los Estados Unidos en la época, ${ }^{3}$ y fue asimilado por el grupo de biblioteconomía de aquel país, como reacción al documento $A$ Nación at RISC [sic], informe publicado en 1983, sobre la situación de la educación norteamericana, que ignoró por completo la contribución que la biblioteca podría darle a la enseñanza (Dudziak, 2003, p. 25; Campello, 2003, p. 31).

Utilizando el concepto de information literacy como base para sus argumentaciones, los actores del área iniciaron un movimiento de reacción en el

3 Las organizaciones que producían esas fuentes ya tenían conocimiento acerca de su baja utilización en las empresas. Esas organizaciones eran representadas por la Information Industries Association, y en 1974, su presidente, Paul Zurkowsky, pidió ante la National Commission on Libraries and Information Science mayor acción del gobierno para capacitar personas que aprendieran habilidades para utilizar las bases de datos que estaban siendo producidas cada vez en mayor cantidad, Zurkowsky utilizó entonces el término information literacy (Behrens, 1994, p. 309). 
que intentaban probar vigorosamente su capacidad de influir en el proceso de enseñanza-aprendizaje. Es necesario recordar que, aunque utilizaran antes el término bibliographic instruction, muchos bibliotecarios estadunidenses que actuaban en las escuelas ya estaban desarrollando acciones pedagógicas más amplias de lo que el referido término implica, y tenían conciencia de que ese trabajo ejercía una influencia positiva en el aprendizaje.

El concepto de alfabetización informativa fue construido por diversas nociones. Una de ellas es la de sociedad de la información, y en su discurso los actores resaltaban las características de ese ambiente de abundancia informativa y de variedad de formas - representadas especialmente por las que resultaban del uso de las tecnologías de información y justificaban la necesidad de crear nuevas habilidades o competencias para trabajar con el ambiente informativo, altamente complejo y mutable. La siguiente declaración contenida en el informe del comité de alfabetización informativa de la ALA, uno de los documentos más citados sobre el asunto, ejemplifica cómo la noción de sociedad de la información está siendo usada en el discurso del movimiento por la alfabetización informativa.

[...] en una sociedad de la información, todas las personas deberían tener derecho a la información que pueda mejorar sus vidas. Viviendo en un ambiente con abundancia de la información, las personas necesitan ser capaces de obtener información específica con el fin de satisfacer sus diversas necesidades personales y profesionales (ALA, 1989).

Se percibe aquí desde el inicio, una fuerte tendencia a hacer un abordaje funcional que se caracteriza por poner énfasis en las habilidades y conocimientos considerados como necesarios para que el individuo funcione adecuadamente en un contexto social que implica el uso frecuente de información.

La noción de tecnología de la información que pasa el concepto de alfabetización informativa es consecuencia natural del asunto anterior. Vivir en la sociedad de la información significa convivir con abundancia y diversidad de información, y la tecnología es el instrumento que facilita el acceso y uso de ese universo informacional amplio y complejo. Sin embargo el carácter instrumental de la tecnología es frecuentemente criticado en el discurso de la alfabetización informativa: la tecnología no es una finalidad, sino un instrumento para mejorar los resultados del aprendizaje (Joint, 2005, pp. 397-398).

La noción de constructivismo también está presente de forma sobresaliente en el concepto de alfabetización informativa, cuya aparición coincide con la época en que bibliotecarios e investigadores de la biblioteconomía y 
de la ciencia de la información se estaban familiarizando con ideas como resource-based learning (Laverty, 2001; Staples, 1997; CASL, 2006; AASL, 1999), aprendizaje independiente (MSACS, 1994 citado por Rader, ${ }^{4}$ 1996), aprender a aprender ${ }^{5}$ (ALA, 1989), aprendizaje a lo largo de la vida (ACRL, 2000), aprendizaje por cuestionamiento (Kuhlthau, 2001), aprendizaje por solución de problemas (Bjorner, 1991, p. 151, citado por Bruce, 1997, p. 27) y pensamiento crítico (Loertscher y Woolls, 1997, p. 338), las cuales fueron incluidas en el discurso de la alfabetización informativa.

Todas esas nociones presentes en el discurso sobre la alfabetización informativa caracterizan la naturaleza interdisciplinar del concepto, y aunque durante algún tiempo hayan sido tratadas con cierta superficialidad, la situación parece estarse modificando. Por ejemplo, una de las nociones recomendadas por el CISSL/IMLS International Research Symposium, ${ }^{6}$ realizado en 2005 , se refería a la necesidad de que se profundizaran conceptos como, por ejemplo, aprendizaje para la toda la vida (lifelong learning). En 2006, Crow (2006, p. 22) amplió el entendimiento del concepto a través del estudio que utilizó la Self Determination Theory (SDT) de E. L. Deci y R. M. Ryan (1985) para analizar la disposición a aprender, que es el principal atributo de la persona que continúa aprendiendo a lo largo de la vida, aun después de terminar su periodo de educación formal. Retomando el tema de la perspectiva educacional para la biblioteconomía, ese tipo de análisis ayuda a entender la situación y prepara caminos para la acción interdisciplinar.

\section{Bases teóricas de la alfabetización informativa en la biblioteconomía}

Coincidiendo con el desarrollo de estudios de usuarios que buscaban comprender el proceso de enseñanza-aprendizaje basado en la búsqueda y en el uso de la información, la utilización del concepto de alfabetización informativa fue significativamente beneficiada con los resultados de esos estudios, los cuales propiciaron el fundamento teórico para su aplicación.

En ese sentido, la investigadora que está teniendo gran influencia en cuestiones de la alfabetización informativa es la norteamericana Carol $\mathrm{Ku}$ hlthau, que desarrolló el modelo que lleva el nombre Information Search Process - ISP (Kuhlthau, 1996, p. 41). Construido a partir de una serie de

4 El texto de Rader no cita la página del texto de la MSACS donde retiró la citación.

5 El concepto "aprender a aprender" se hizo conocer a partir del documento La educación encierra un tesoro, preparado para la UNESCO, por la Comisión Internacional sobre la Educación para el siglo XXI, coordinada por Jacques Delors.

6 O CISSL/IMLS International Research Symposium fue promovido por el Center for International Scholarship in School Libraries, de la Rutgers University, N. J, junto al Institute of Museum and Library Service (EUA), en abril de 2005. 
estudios, el modelo se basa en la teoría constructivista del aprendizaje y en la dimensión afectiva, y es muy utilizado por los investigadores del área de ciencia de la información. Eso se debe al hecho de que Kuhlthau trató de forma profunda el proceso de búsqueda de información en un ambiente escolar, y a que presentó un modelo que enfatiza la comprensión del proceso de investigación, cuyo espacio natural es la biblioteca y el cual propicia oportunidades para el desarrollo de diversas habilidades relacionadas con la información.

El modelo abarca tres aspectos del proceso de búsqueda de la información; a saber: los pensamientos que ocurren durante el proceso (dimensión cognitiva), los sentimientos que acompañan típicamente la evolución del pensamiento (dimensión afectiva) y las acciones de buscar y usar fuentes de información (dimensión física), los cuales se componen de seis fases: inicio del trabajo, selección del asunto, explotación del foco, definición del foco, recopilación de informaciones, presentación de los resultados y la evaluación del proceso (Kuhlthau, 1996, pp. 33-56).

Otra vertiente de estudios que apoyan teóricamente la alfabetización informativa es aquella que busca identificar y comprender las características de la persona capacitada en el uso de la información. Dos autoras son representativas de esa vertiente: Christina Doyle y Christine Bruce, quienes realizaron sus investigaciones a partir de la década de 1990.

Realizado en el ámbito del National Fórum on Information Literacy, el estudio de Doyle (1992) utilizó la técnica de Delphi, para alcanzar un consenso de 136 participantes con relación a la definición de alfabetización informativa y a los atributos de la persona competente para trabajar con la información; llegó al siguiente resultado: "alfabetización informativa es la habilidad para acceder, evaluar y usar la información de una variedad de fuentes" (Doyle, 1992, p. 4). La definición se amplía incluyendo diez atributos:

La persona competente en información es aquella capaz de: reconocer la necesidad de información; reconocer que la información cuidada y completa es la base para tomar decisiones inteligentes; formular preguntas basadas en la necesidad de la información; identificar fuentes potenciales de información; desarrollar estrategias de búsqueda adecuadas; acceder a fuentes de información, incluso electrónicas; evaluar información, organizar información para aplicaciones prácticas e integrar nueva información al cuerpo de conocimientos existente; usar información para pensar críticamente y para solucionar problemas (Doyle, 1992, p. 4).

Bruce (1997), a su vez, utilizó la fenomenología para crear un modelo relacional, que ve la alfabetización informativa como un fenómeno experimentado 
por personas que interactúan con el universo informacional; esto es, que usan la información de forma competente. La alfabetización informativa es descrita con base en las diversas maneras en que las personas la hayan vivido. Las concepciones de ahí resultantes no representan atributos individuales, es decir conocimientos, habilidades y actitudes que el individuo necesita dominar para ser competente en el uso de la información, sino las diferentes relaciones que se establecen entre el usuario y la información. En esa perspectiva, los atributos asumen una posición secundaria y, lo más importante, son las diferentes maneras de concebir lo que significa ser competente en determinada situación; en este caso, el modo como la persona interactúa con la información. Las siete concepciones o experiencias que incluyen el fenómeno de la alfabetización informativa de Bruce (1997, pp. 117-151) son: experiencia en la tecnología de la información, experiencia en las fuentes de información, experiencia en el proceso de información, experiencia en el control de la información, experiencia en la construcción del conocimiento, experiencia acerca de la extensión del conocimiento y experiencia en el saber.

Los trabajos de esas tres autoras, (Kuhlthau, Doyle y Bruce) anteriormente sintetizados, pueden ser considerados representativos del conjunto de investigaciones que se están realizando para obtener una mejor comprensión acerca de la cuestión de la alfabetización informativa, lo cual permitiría hacer una definición más cuidada sobre el papel pedagógico que desempeña el bibliotecario. La consolidación de los fundamentos teóricos acerca de la alfabetización informativa constituyen el factor más importante que justifica y estimula al bibliotecario a ocupar cada vez más espacio en el proceso de ayudar a las personas a aprender y a producir conocimiento con base en la información.

Se pueden observar avances en investigaciones más recientes que buscan demostrar los resultados del aprendizaje a través de la búsqueda y el uso de la información. En esa línea se incluye el trabajo de Todd (2005) quien estudió un grupo de 43 alumnos de enseñanza secundaria comprometidos con un proyecto de investigación cuyo objetivo era entender el proceso de construcción del conocimiento que incluye el uso continuado y frecuente de fuentes de información variadas y la verificación y el impacto que tienen un ambiente de aprendizaje positivo y facilitador, en el cual los alumnos tenían la oportunidad de exponer sus ideas y descubrimientos, los criterios de evaluación eran explícitos y, principalmente, había una mediación adecuada de profesores y bibliotecarios en cada fase del proceso dentro de un clima colaborativo. La investigación reveló resultados positivos relacionados con el aprendizaje de los estudiantes.

Se verifica entonces que las acciones de alfabetización informativa están siendo favorecidas por investigaciones que, cada vez profundizan más en las 
cuestiones de aprendizaje y propician en los bibliotecarios mejores condiciones para trabajar de forma colaborativa con los profesionales de la educación.

\section{Práctica de la alfabetización informativa}

Junto a la realización de estudios académicos, empezaron también a crearse diversos tipos de instrumentos que posibilitaron la aplicación más eficiente de programas de alfabetización informativa en las escuelas.

Los parámetros que definen las habilidades informativas han sido creados por instituciones importantes y entidades educacionales, como la American Association of School Librarians (AASL) y la Association of College and Research Libraries (ACRL). Tales parámetros sistematizan las habilidades informativas y definen estándares que deben alcanzar los programas de alfabetización informativa (AASL/1998; ACRL, 2000). También la International Federation of Library Associations and Institutions (IFLA) lanzó, en 2006, las Guidelines on information literacy for lifelong learning (Lau, 2006), una herramienta práctica para profesionales que deseen iniciar o evaluar programas de alfabetización informativa.

Otro tipo de instrumento que auxilia en la práctica de la alfabetización informativa son los modelos que sistematizan el proceso de investigación. Se crearon muchos de esos modelos para auxiliar a los estudiantes a recorrer las diversas etapas del proceso, aprendiendo a investigar de manera disciplinada. Uno de los más conocidos es el Big6, desarrollado por los estadunidenses Michael B. Eisenberg y Robert E. Berkowitz en 1987, estructurado en seis pasos: definición del problema de información; identificación de las informaciones necesarias; elección e identificación de las fuentes; interpretación y síntesis del contenido, y evaluación del producto y del proceso (What, 2004). Otro ejemplo es el instrumento creado por el autor británico Michael Marland, que también está estructurado en pasos que deben seguirse durante el proceso de investigación y que están presentados en forma de preguntas: “¿Qué necesito hacer? ¿Dónde puedo ir? ¿Dónde consigo información? ¿Qué recursos debo usar? ¿Cómo debo usar los recursos? ¿Qué debo registrar? ¿Tendré la información que necesito? ¿Cómo debo hacer la presentación? ¿Qué obtuve? (Marland, 1981 citado por Alves, 1999).

La aplicación de programas de alfabetización informativa en bibliotecas escolares condujo naturalmente hacia la necesidad de evaluar. Los actores han mostrado interés en medir el proceso de aprendizaje por la búsqueda y el uso de la información, identificando evidencias no solamente en relación con las ventajas del aprendizaje constructivista, sino también con el papel desempañado por el bibliotecario en ese proceso, cuando trabaja junto a los profesores. 
La creación del SLIM (School Library Impact Measure) por un grupo de investigadores del CISSL (Center for International Scholarship on School Libraries) constituye una estrategia para verificar y comprobar lo que aprendieron los estudiantes en relación con la información. Y fue elaborado para evaluar proyectos escolares basados en el aprendizaje por cuestionamiento (inquiry learning), en el que los estudiantes se involucran activamente con fuentes de información diversas y generalmente confrontadoras, para descubrir nuevas ideas, construir nuevas comprensiones y desarrollar puntos de vista y perspectivas personales. La metodología del SLIM se fundamenta en la reflexión del alumno sobre su aprendizaje, realizada en tres etapas del proceso: al inicio, a la mitad y al final, cuando se completa el trabajo. Ese tipo de metodología permite verificar cambios en el aprendizaje y aporta aclaraciones para mejorar la mediación, a la vez que proporciona evidencias respecto al papel pedagógico que desempeña la biblioteca (Todd; Kuhlthau; Heinström, 2005, p. 5).

\section{Perspectivas del concepto de alfabetización informativa}

Se observa que las investigaciones y prácticas descritas anteriormente ayudaron a ampliar la comprensión sobre la alfabetización informativa mucho más que los extensos intentos de definirla que se están emprendiendo (Behrens, 1994; Bruce, 1997; Bawder, 2001), en general poco útiles, en la medida en que se limitan a presentar las ideas de varios autores y mantienen una circularidad y repetición constantes. En muchos de esos trabajos, la alfabetización informativa fue tratada como un asunto de interés exclusivo para los bibliotecarios, así que eran pocos los autores que demostraban conciencia acerca de la característica interdisciplinar del concepto, especialmente de su relación con la alfabetización en general.

En algunos textos la relación de la alfabetización informativa con la alfabetización se fue profundizando, como sucedió con el de Langford (1998), quien defendió la idea de la alfabetización como un contínuum, argumentando que el concepto evolucionó en la medida en que las necesidades de conocimiento de la sociedad se modificaron, y que han seguido adquiriendo diferentes facetas que en la actualidad están relacionadas de forma estrecha con el uso de la tecnología de la información. En ese sentido, la alfabetización informativa no sería un objetivo fijo a ser alcanzado, sino un contínuum de habilidades, familiaridad y eficiencia relativas al uso de la información, representado por grados crecientes de dominio.

La autora consideró que mientras la alfabetización informativa no sea considerada por los educadores como parte natural de las prácticas pedagógicas 
en general, y sea tratada por los bibliotecarios como algo aislado, es poco probable que sea incorporada como práctica en la escuela. Según Langford (1998), el discurso de la alfabetización informativa debería eliminar el argot bibliotecario, buscar objetivos comunes entre los educadores y tomar en cuenta que los bibliotecarios no serían los únicos interesados en los asuntos relacionados con el aprendizaje por medio de la información. Así, la noción de la alfabetización como un contínuum debería ser privilegiada y las prácticas informativas tendrían que irse incorporando naturalmente a las estrategias didácticas y no ser sólo incluidas como un "tópico" más del currículo.

La mayoría de las iniciativas y textos analíticos sobre alfabetización informativa enfatizan su dimensión funcional, de atributo personal, y se refieren a la posesión individual de habilidades para usar la información, de la misma forma que la alfabetización en su dimensión individual se considera como un conjunto de habilidades de lectura y escritura que la persona posee (Soares, 2007, pp. 30-33). En esa dimensión, las habilidades informativas están consideradas como indispensables para que el individuo se integre adecuadamente en la sociedad de la información y sobreviva en el trabajo, en la vida diaria, etcétera; esto es, se presupone la adaptación del individuo a las circunstancias de la sociedad en la que vive.

Más escasos son los textos en que las habilidades informativas no están consideradas como 'neutras' — capacidades que deberán ser usadas en prácticas sociales cuando sea necesario - sino como un conjunto de prácticas socialmente construidas, configuradas por procesos sociales más amplios, y responsables por el reforzamiento o el cuestionamiento de valores, tradiciones y estándares de poder presentes en el contexto social (Soares, 2007, p. 35). Pawley (2003, p. 10) fue una de las autoras que reforzaron la necesidad de contar con un discurso más crítico para la alfabetización informativa, que se aleje de la tradición del área, la cual generalmente utiliza un lenguaje gerencial y direccionado hacia cuestiones técnicas y administrativas que impiden cuestionar los valores de su práctica. Sugirió que los actores se involucren en reflexiones y análisis críticos que posibiliten la explicitación de cuestiones de investigación en las que la unidad de análisis no sea la organización ni el individuo, sino la sociedad. La definición estandarizada de alfabetización informativa no muestra el carácter ideológico y las relaciones de poder involucradas en el asunto e impide, según la autora, que los puntos esenciales sean discutidos. En lugar de negociar conceptos formales y técnicas sobre la mejor manera de transmitir habilidades previamente definidas, los practicantes deberían discutir qué es, fundamentalmente, lo que están intentando realizar al involucrarse en prácticas de alfabetización informativa. Ése es el desafío que obliga a los practicantes a que se pregunten también el "cómo", para responder lo cual deben buscar referencias en la educación, en la epistemología, en 
la ética, en la política y en la teoría social (Pawley, 2003, p. 11). Hay preguntas básicas que necesitan ser planteadas: "alfabetización informativa ¿para qué y para quién?”, “¿En qué circunstancias sociales e institucionales ocurre?”, "¿Qué consecuencias tiene para la distribución de bienes sociales y culturales en la sociedad como un todo?" Los actores en todo esto deben reconocer que las relaciones de poder constituidas social e históricamente y basadas, por ejemplo, en cuanto a clase, raza, género y edad, pueden influir en el pensamiento y las definiciones sobre el conocimiento. Un abordaje crítico del conocimiento debe indagar cómo está definido éste, por quién, hacia quién, y cómo esos procesos contribuyen a reproducir, mediar o transformar las relaciones de poder. Pawley (2003, p. 11) consideró que el lenguaje desempeñaba un papel clave en la construcción de la conciencia y critica el lenguaje que se está usando para describir y discutir la alfabetización informativa. Propuso que se aclare sobre ¿quién habla a favor, en qué términos y en el interés de quién más? La propuesta de la autora es "desnaturalizar lo natural", eliminar la naturaleza inherente y neutra del discurso de la alfabetización informativa y cuestionar lo que ha sido determinado previamente. Aclara que no se trata solamente de una cuestión de curiosidad intelectual, sino de buscar democratizar las relaciones sociales, políticas, económicas y culturales, en un proceso de emancipación (Pawley, 2003, p. 11).

Así, la autora citada les recomendó a los actores bibliotecológicos que pusieran el uso de la lengua críticamente bajo escrutinio, lo que les permitiría que se fijaran en el contenido y en el contexto de la información, incluyendo la acción su producción, su uso y la acción de re-contextualizar. Es necesario entender que "usos y usuarios de la información" o "textos y lectores" no pueden ser tratados como grupos que van a manipularse, sino como comunidades discursivas y comunidades de práctica; esto es, comunidades que tienen también condiciones de producir información y no solamente de consumirla. La enseñanza de habilidades o competencias para el uso de la información debería, por lo tanto, ser planeada con base en las necesidades reales de las comunidades, de los grupos y de las personas. Eso sucederá cuando los actores trabajen en colaboración con otras agencias que suministren información para grupos o comunidades, y abran caminos para concebir una alfabetización informativa orientada hacia la dimensión social. Pawley concluyó que los actores interesados deben empezar a describir lo que realmente sucede durante el tumultuoso proceso de investigación (uso de información) al contrario de lo que piensan que ocurre y que de ese modo tendrán mejores condiciones para ejercer su función pedagógica.

Tuominen, Savolainen y Talja (2005), por su vez, sorprendidos con el enorme y creciente número de publicaciones sobre alfabetización informativa 
que ha surgido en las últimas tres décadas, criticaron también el abordaje funcional que domina el área. Según esos autores, la alfabetización informativa hoy constituye principalmente una noción práctica y estratégica, utilizada para guiar las acciones de los bibliotecarios hacia la enseñanza de habilidades informativas, cuando aún se carece de estudios empíricos sobre el tema. Los textos sobre el asunto mantienen un tono prescriptivo, y resaltan el conjunto de habilidades necesarias para poder vivir en la sociedad contemporánea. Capacidades o competencias que son consideradas como atributos personales que pueden ser enseñados y evaluados, independientemente de los contextos en los que son utilizados. Tuominen, Savolainen y Talja (2005) defendieron en ese entonces el abordaje de la alfabetización informativa como práctica "socio-técnica", y recomendaron la realización de estudios basados en una concepción naturalista del trabajo y del aprendizaje, pues, según ellos, los autores del área, hasta el momento, no se han preocupado por la necesidad de entender cómo las personas interactúan con otras y con los equipos informativos en el ambiente específico en que se mueven. Así, es necesario conocer los dominios del conocimiento, las organizaciones y las tareas prácticas en las que estas habilidades o capacidades informativas son utilizadas. Según los autores citados, la alfabetización informativa sólo avanzará en caso de que los actores entiendan la inter-acción existente entre las tecnologías de la información y los procesos de aprendizaje en áreas específicas, para lo cual hay que realizar estudios empíricos que analicen la manera en que determinada comunidad utiliza los diversos instrumentos conceptuales, culturales y técnicos para acceder y usar documentos impresos y digitales, y para evaluar y crear conocimiento. La naturaleza sociocultural es central para los autores mencionados, quienes proponen un nuevo abordaje para la alfabetización informativa, partiendo del principio de que debería fijarse principalmente en las posibilidades de los grupos y las comunidades para que cultiven sus acciones de información, y para encontrar estrategias de apoyo para sus interacciones con las tecnologías de la información.

Buscando consolidar la profusión de definiciones y descripciones de alfabetización informativa que surgieron desde la época en que el término empezó a integrar el vocabulario del área de la biblioteconomía, Owusu-Ansah (2005) produjo un trabajo aclarador y concluyó que las controversias existentes entre ellas son irrelevantes y que prevalecen los puntos en común. El autor apoyó su opinión (de que hay claridad suficiente en el concepto de alfabetización informativa) en la visión de Saracevic (1999, p. 1054), según la cual los conceptos son definidos de manera más adecuada en la medida que van siendo investigadas las manifestaciones, los comportamientos y los efectos del fenómeno que representan. El autor consideró que las manifestaciones 
de la alfabetización informativa ya fueron identificadas en las diversas listas de habilidades o competencias, en las definiciones de comportamientos y en las descripciones del proceso, presentadas en la literatura hasta el momento, lo cual permite que se alcance concordancia con relación a los siguientes aspectos: 1) hay deficiencias en la formación del usuario con relación al uso de la información, principalmente a la información electrónica; 2) el papel de la biblioteca es ayudar en esa formación; 3) en el área de la educación esa formación puede ser articulada como objetivo educacional e integrar éste al currículo; 4) esa formación puede proveer conceptos e instrumentos para que los alumnos avancen de forma autónoma en su aprendizaje. Owusu-Ansah (2005) sugirió que el momento era propicio para que se trabajaran formas de mejorar las capacidades de los estudiantes y éstos pudieran aprender, con base en la información, a explotar el papel de la biblioteca, y a determinar la legitimidad de la participación del bibliotecario en el proceso, así como el grado en que éste desea participar. Consideró que, comprendiendo lo que los bibliotecarios identifican como su contribución singular para la alfabetización informativa, es posible buscar maneras de ayudar a las personas en el proceso de aprender con base en la información.

\section{La evolución de la investigación sobre alfabetización informativa}

Analizando el trayecto de los estudios sobre alfabetización informativa, Arp y Woodard (2002, p. 129), se basaron en el estudio de Keresztesi (1982, pp. 13-21), que identificó tres fases en el desarrollo de una asignatura. El análisis de Arp y Woodard (2002) concluyó que los estudios sobre la alfabetización informativa se encuentran en la fase en que están pasando de un periodo pionero (caracterizado por esfuerzos para obtener reconocimiento, atención y persuadir), que ocurrió principalmente durante la década de 1980, hacia una fase intermedia de elaboración y proliferación (en la década de 1990), en la que el número de investigadores y de investigaciones se amplía, los asuntos estudiados son más complejos y se subdividen, hay más preocupación por la metodología, la terminología se diversifica y aparecen confusiones semánticas. El próximo paso sería el de la consolidación.

Loertscher y Woolls (1997) sintetizaron los resultados de las investigaciones, en términos de los conocimientos hasta aquel momento obtenidos y concluyeron que los estudios revelaban una relación estrecha entre la alfabetización informativa y las teorías constructivistas de aprendizaje, en especial el aprendizaje por cuestionamiento, aunque reconocieron que esa estrategia didáctica aún no era la más usada en las escuelas. Sugirieron que el bibliotecario debería ser más realista y buscar formas de trabajar en el contexto de 
otras estrategias para implementar actividades de alfabetización informativa. Los autores reconocieron que había un largo camino por recorrer, ya que las investigaciones sobre aprendizaje por medio de la información aún no estaban causando impacto significativo en la práctica bibliotecaria.

En 1999, analizando trabajos originados en los Estados Unidos y Australia, Bruce (1999) los categorizó en: 1) investigaciones en la práctica, que consisten en analizar programas de alfabetización informativa buscando su perfeccionamiento; 2) investigaciones aplicadas, que estudian cuestiones que ayudarán en la implementación de programas tales como, necesidades y perfiles de usuarios, y evaluación de programas; 3 ) investigaciones puras que, utilizando métodos variados, buscan comprender el fenómeno en sus variadas facetas, y se dedican principalmente a entender cómo las personas aprenden con la información o cómo viven la experiencia de la alfabetización informativa.

Un año más tarde, la misma autora (Bruce, 2000, pp. 1-2) realizó otro análisis del estado del arte de la investigación sobre alfabetización informativa, y la organizó en cuatro etapas de desarrollo, a saber: 1) etapa de los precursores (década de 1980), representada por estudios sobre instrucción bibliográfica y otros, como el de Kuhlthau (1996) quien definió la alfabetización informativa como una manera de aprender; 2) etapa experimental (1990 hasta $1995)$ representada por estudios como el de Christina Doyle (1992), que condujo las definiciones de alfabetización informativa que hasta hoy influyen en la práctica; 3 ) etapa exploratoria (1995-1999), cuando hubo un aumento en el número de investigaciones que utilizaron enfoques metodológicos variados; 4) etapa de expansión (2000-), cuando se visualizó una tendencia hacia la realización de investigaciones aplicadas y en la práctica, de acuerdo con la categorización hecha en su estudio anterior (Bruce, 1999). En realidad, relatos de investigaciones que la autora denominó "puras" han aparecido con frecuencia en la literatura, recomendando una comprensión más profunda del proceso de aprendizaje mediante el uso de la información (Limberg, 1999; Harada, 2002; Todd, 2005).

Se puede decir que el esfuerzo realizado hasta el momento -sea en la forma de estudios académicos que generaron modelos de alfabetización informativa, de estructuras para la enseñanza de habilidades, de listas de capacidades por alcanzar o de criterios de evaluación- ha sido necesario para comprender el concepto que permite el avance de todo este asunto. Según Arp y Woodard (2002, p. 130), los esclarecimientos que ocurrieron después de cada debate sobre el tema han ayudado a aclararles el concepto, no sólo a los bibliotecarios, sino también a los profesionales de otras áreas, y han ayudado a generar una perspectiva más clara sobre el papel que juega la biblioteca actualmente y 
sobre lo que es la alfabetización informativa. Según las autoras, ese proceso debe continuar. Los debates en torno al concepto de alfabetización informativa son normales y reflejan las dificultades que han ocurrido siempre que un área amplía sus intereses. Capurro (citado por Matheus ${ }^{7}, 2005$, pp. 153-154) identificó esta tendencia con relación a la ciencia de la información, argumentando que la ampliación de intereses en un determinado campo del conocimiento aumenta su interdisciplinaridad y aumenta las dificultades para que se alcancen consensos con respecto a cuestiones básicas.

\section{Conclusión}

Pasada la euforia inicial del surgimiento del término, los actores del área bibliotecaria ya han percibido la complejidad de la alfabetización informativa y buscan su mejor comprensión. Aceptan que la alfabetización informativa puede constituir la estructura conceptual que legitime su papel educativo. Esto ocurre cuando las tareas técnicas del bibliotecario se transforman en rutina, en virtud de la utilización de la tecnología (O'Connor, 2009, p. 286) y en un momento en que la profesión se encuentra frente al desafío de darle más visibilidad a su función educativa y de asumir la responsabilidad de ayudar a las personas a manejar y a trabajar con el aparato informacional para aprender.

El hecho es que esa relación con el aprendizaje produce consecuencias para la práctica profesional.

El cuadro siguiente sintetiza los cambios que la implantación de programas de alfabetización informativa implicará en la práctica educativa del bibliotecario.

\begin{tabular}{|ll|}
\hline \multicolumn{1}{|c|}{ Cambios en la práctica educativa del bibliotecario } \\
\hline Oferta aleatoria de actividades $\longrightarrow$ & Oferta planeada de actividades \\
Intuitiva $\longrightarrow$ & Basada en teorías, conocimientos, \\
& evidencias \\
Responsabilidad del bibliotecario $\longrightarrow$ & Responsabilidad de toda la escuela \\
Esporádica $\longrightarrow$ Constante y gradual \\
Limitada a la biblioteca $\longrightarrow$ Integrada al currículo \\
Enfocada en actividades $\longrightarrow$ Enfocada en habilidades \\
Evaluación de las actividades $\longrightarrow$ Evaluación del aprendizaje \\
\hline
\end{tabular}

En síntesis, la implementación de programas de alfabetización informativa exige prácticas planeadas, fundamentadas en evidencias y teorías que

7 Matheus no especifica la fuente de la idea de Capurro. 
constan de actividades sistemáticas y secuenciales, e involucran no sólo al bibliotecario, sino que son compartidas entre otros miembros del equipo escolar.

El objetivo es desarrollar competencias o habilidades, y por lo tanto, el foco se desplaza de las actividades de la biblioteca hacia la capacitación de los alumnos, lo que lleva a la necesidad de instaurar procesos evaluativos orientados a verificar el aprendizaje. El apoyo al usuario no es individual sino colectivo, e involucrando también a grupos de estudiantes, y no solo a individuos.

La emergencia del concepto de alfabetización informativa, que está acompañada por referencias teóricas más elaboradas y por descripciones de experiencias prácticas exitosas, constituye, por lo tanto, una oportunidad para que la clase bibliotecaria reflexione sobre su papel educativo; $y$, a partir de esta reflexión, implemente acciones y desarrolle investigaciones que concreten este papel.

\section{BiBLIOGRAFIA}

Alves, M. P., "Biblioteca escolar: tecnologias de informação e currículo”, en Liberpolis, n. 2, pp. 69-80, 1999.

American Association of School Librarians, Position statement on resource based instruction: role of the school library media specialist in reading development, Chicago, 1999, disponible en: < http:// www.ala.org/ala/mgrps/divs/aasl/aaslissues/positionstatements/ readingdevelopment.cfm >, acesso el: 20 set. 2009.

American Association of School Librarians/ Association for Educational Communications and Technology, Information power: building partnerships for learning, Chicago, ALA, 1998, disponible en: http://www.ala.org/ala/mgrps/divs/aasl/aaslproftools/informationpower/InformationLiteracyStandards_final. pdf, acesso el 20 set. 2009.

American Library Association. Presidential Committee on Information Literacy, Final report, Chicago, 1989, disponible en: < http:// www.ala.org/ala/mgrps/divs/acrl/publications/whitepapers/ presidential.cfm>, acesso el 20 set. 2009.

Arp, L.; Woodard, B. S., "Recent trends in information literacy and standards", en Reference \& User Service Quarterly, v. 42, núm 2, pp. 124-132, 2002.

Association of College And Research Libraries, Information literacy competency standards for higher education, Chicago, IL, 2000, [tradução em espanhol], disponible en < http://www.ala.org/ala/ mgrps/divs/acrl/standards/informationliteracycompetency.cfm >. acesso el 20 set. 2009. 
Bawder, D., "Information and digital literacies: a review of concepts", en Journal of Documentation, v. 57, núm. 2, pp. 218-259, 2001.

Behrens, S. J., "A conceptual analysis and historical overview of information literacy", en College E Research Libraries, v. 55, num 4, pp. 309-322, 1994.

Bjorner, S., "The information literacy curriculum: a working model”, IATUL Quarterly, v. 5, núm. 2, pp.150-169, 1991.

Bruce, C., "Information literacy: an international review of programs”, en Lianza Conference, 1999, Auckland, Papers... Auckland, Lianza, 1999.

Bruce, C., The seven faces of information literacy, Adelaide, Auslib, 1997, 203p.

Campello, B., "O movimento da competência informacional: uma perspectiva para o letramento informacional”, Ci. Inf., Brasília, v. 32, núm.3, pp. 28-37, 2003.

Canadian Association for School Libraries, Guidelines for effective school library programs: a position statement on effective school library programs in Canada, disponible en: < http://www.cla.ca/ casl/guidelines.html >, acesso el 20 set. 2009.

Capurro, R.; Hjorland, B., "The concept of information”, en Annual Review of Information Science and Technology, v. 37, pp. 343-411, 2003 a.

Craver, K. W., "The changing instructional role of the high school library media specialist: 1950-84", en School Library Media Quarterly, v. 14, núm. 4, 183-191, 1986.

Crow, S. R., "What motivates a lifelong learner?", en School Libraries Worldwide, v. 12, núm. 1, pp. 22-34, 2006.

Deci, E. L.; Ryan, R. M., Intrinsic motivation and self-determination in buman behavior, New York: Plenum, 1985 citado por Crow, S. R. "What motivates a lifelong learner?" en School Libraries Worldwide, v. 12, núm. 1, pp. 22-34, 2006.

Doyle, C., Outcome measures for information literacy within the national education goals of 1990: final report of the National Forum on Information Literacy, Summary of findings, Washington, DC, US Department of Education, 1992, [ERIC document no; ED 351033], disponible en http://www.eric.ed.gov/ERICDocs/data/ ericdocs2sql/content_storage_01/0000019b/80/13/2c/6e.pdf, acesso el 20 set. 2009.

Dudziak, E.A., "Information literacy: princípios, filosofia e prática", en Ci. Inf., v. 32, núm. 1, pp. 23-35, 2003.

Frank, D. G. et al., The changing nature of reference and information services: predictions and realities, en Reference $\mathcal{E}$ User Services Quarterly, v. 39, núm 2, pp. 151-157, 1999.

Harada, V., "Personalizing the information search process: a case study of journal writing with elementary age students", en School Library Media Research, v. 5, 2002, disponible en http://www.ala. org/ala/aasl/aaslpubsandjournals/slmrb/slmrcontents/volume52002/harada.htm, acesso el 20 set. 2009. 
Joint, N., “Traditional bibliographic instruction and today's information users", en Library Review, v. 54, núm. 7, pp. 397-402, 2005, disponible en http://www.emeraldinsight.com/Insight/viewPDF. jsp?Filename $=$ html/Output $/$ Published/EmeraldFullTextArticle/Pdf/0350540701.pdf, acesso el 20 set. 2009.

Keresztesi, M., "The science of bibliography: theoretical implications for bibliographic instruction”, en Oberman, C.; Strauch, K. Theories of bibliographic education: designs for teaching, New York, Bowker, 1982, pp. 2-26.

Kuhlthau, C. C., "Rethinking libraries for the information age school: vital roles in inquiry learning" en IASL CONFERENCE, 2001, Auckland. Keynote paper:.. Auckland, 2001, disponible en < http:// www.iasl-online.org/events/conf/keynote-kuhlthau2001.html>, acesso el 20 set. 2009.

Kuhlthau, C. C., Seeking meaning: a process approach to library and information services, Norwood, NJ, Ablex, 1996, 199p.

Langford, L., "Information literacy? Seeking clarification", en School Libraries Worldwide, v. 4, núm. 1, pp. 59-72, 1998.

Lau, J., Guidelines on information literacy for lifelong learning, IFLA, 2006, disponible en < http://archive.ifla.org/VII/s42/pub/ILGuidelines2006.pdf>, acesso el 20 set. 2009.

Laverty, C., Resource-based learning, Ontario: Queen's University, 2001, disponible en http://ibrary.queensu.ca/inforef/tutorials7rbl/index.htm, acesso el 29 jan. 2006.

Limberg, L., "Experiencing information seeking and learning: a study of the interaction between two phenomena", en Information Research, v. 5, núm. 1, Oct. 1999, disponible en http://informationr.net/ir/5-1/paper68.html, acesso el 20 set. 2009.

Loertscher, D. V.; Woolls, B., "The information literacy movement of the school library media field: a preliminary summary of the research", en Lighthall, L.; Haycock, K. Information rich but knowledge poor?: emerging issues for schools and libraries worldwide, Seattle, IASL, 1997, pp. 337-358, disponible en http://witloof.sjsu. edu/courses/250.loertscher/modelloer.html, acesso el 20 set. 2009.

Marland, M. (Ed.), Information skills in the secondary curriculum: the recommendations of a working group sponsored by the British Library and the Schools Council, London: Methuen Educational, 1981 citado por Alves, M. P. "Biblioteca escolar: tecnologias de informação e currículo”, en Liberpolis, núm. 2, pp. 69-80, 1999.

Martucci, E. M., "Revisitando o trabalho de referência: uma contribuição teórica para a abordagem interpretativa de pesquisa", Perspectivas em Ciência da Informação, Belo Horizonte, v. 5, núm. 1, pp. 99-115, 2000.

Matheus, R. F., "Rafael Capurro e a filosofia da informação: abordagens, conceitos e metodologias de pesquisa para a ciência da informação", en Perspectivas em Ciência da Informação, Belo Horizonte, v. 10, núm. 2, pp. 140-165, 2005. 
Middle States Association of Colleges and School. Commission on Higher Education, Information literacy: lifelong learning in the Middle States Region, a summary of two symposia, Philadelphia: Middle States Association, 1995 citado por Rader, H. B. Educating students for the information age: the role of the librarian, en China-United States Library Conference, 1., 1996, Beijing, Conference reports... Beijing, National Library of China, 1996, disponible en http://darkwing.uoregon.edu/ felsing/ala/rader.html, acesso el 20 set. 2009.

O'Connor, L., "Information literacy as professional legitimation: the quest for professional jurisdiction", en Library Review, v. 58, núm. 4, pp. 272-289, 2009.

Ortega y Gasset, José, Misión del bibliotecario y otros ensayos afines, 2. ed. Madrid, Ediciones de Revista de Occidente, 1967, 183p, disponible en http://65.99.205.133/ dgbcona/publicaciones/mision_bibliotecario.pdf, acesso el 20 set. 2009.

Owusu-Ansah, E. K., "Debating definitions of information literacy: enough is enough!", en Library Review, v. 54, núm. 6, pp. 366-374, 2005.

Pawley, C., Information literacy: a contradictory coupling, en Library Quarterly, v. 73, núm. 4, pp. 422-431, 2003.

Rader, H. B., "Educating students for the information age: the role of the librarian", en CHINA-UNITED STATES LIBRARY CONFERENCE, 1., 1996, Beijing, Conference reports... Beijing: National Library of China, 1996.

Shera, J. H., Knowing books and men: knowing computers, too, Littleton, Colo., Libraries Unlimited, 1973, 363p.

Soares, M., "Língua escrita, sociedade e cultura; relações, dimensões e perspectivas", en Alfabetização e letramento, São Paulo, Contexto, 2007, pp. 27-45.

Staples, M., Making the most of resource-based learning, The British Library Board/ Library Information Technology Centre, South Bank University, 1997.

Stripling, B. K., Quality in school library media programs: focus on learning, Library Trends, v. 44, núm. 3, pp. 631-656, 1996.

Todd, R. J.; Kuhlthau, C. C.; Heinström, J. E., School Library Impact Measurement (SLIM): a toolkit and handbook for tracking and assessing student learning outcomes of guided inquiry throught the school library, CISSL, Rutgers University, NJ, 2005, disponible en < http://cissl.scils.rutgers.edu/guided_inquiry/docs/SLIM_toolkit. pdf $>$, acesso el 20 set. 2009.

Todd, R., School librarians and educational leadership: productive pedagogy for the information age school, en LEE, S. et al. (Ed.). IASL Reports 2005: information leadership in a culture of change, Erie, PA, International Association of School Librarianship, 2005.

Tuominem, K.; Savolainen; R.; Talja, S., "Information literacy as a sociotechnical practice”, Library Quarterly, v. 75, núm. 3, pp. 329$344,2005$. 
Tyckoson, D., What we do: reaffirming the founding principles of reference services, Reference Librarian, núm. 59, pp. 3-13, 1997.

What is the Big6 ${ }^{\mathrm{TM}}$ ?, Big6 Associates, 2004, disponible en < http:// www.big6.com/>, acesso el 20 set. 2009.

$\infty$ 